\title{
Correlations between structural and electronic properties in the filled skutterudite $\mathrm{Sm}_{\mathrm{y}}\left(\mathrm{Fe}_{\mathrm{x}} \mathrm{Ni}_{1-\mathrm{x}}\right)_{4} \mathrm{Sb}_{12}$
}

Cristina Artini 1,2,*, Gilda Zanicchi 1,3, Giorgio Andrea Costa 1,4, Maria Maddalena Carnasciali $^{1,3}$,Carlo Fanciulli ${ }^{5}$, Riccardo Carlini ${ }^{1,3}$

${ }^{1}$ Department of Chemistry and Industrial Chemistry - University of Genoa, Via

Dodecaneso 31, 16146 Genova, Italy

${ }^{2}$ CNR-IENI, Via De Marini 6, 16149 Genova, Italy

${ }^{3}$ INSTM - Interuniversitary Consortium of Science and Technology of Materials Genova Research Unit, Via Dodecaneso 31, 16146 Genova, Italy

${ }^{4}$ CNR-SPIN Genova, Corso Perrone 24, 16152 Genova, Italy

${ }^{5}$ CNR-IENI, Corso Promessi Sposi 29, 23900 Lecco, Italy 


\section{Rietveld refinement plots}

In this file all the Rietveld plots obtained after the last refinement cycle are reported. The dotted and the continuous line are the experimental and the calculated diffractogram, respectively; the lower line is the difference curve; the vertical lines indicate the position of the expected peaks.

According to the $\% \mathrm{Fe}$ content with respect to the total $(\mathrm{Fe}+\mathrm{Ni})$ content, samples are named $\mathrm{Fe} 100, \mathrm{Fe} 90$, and so on. 


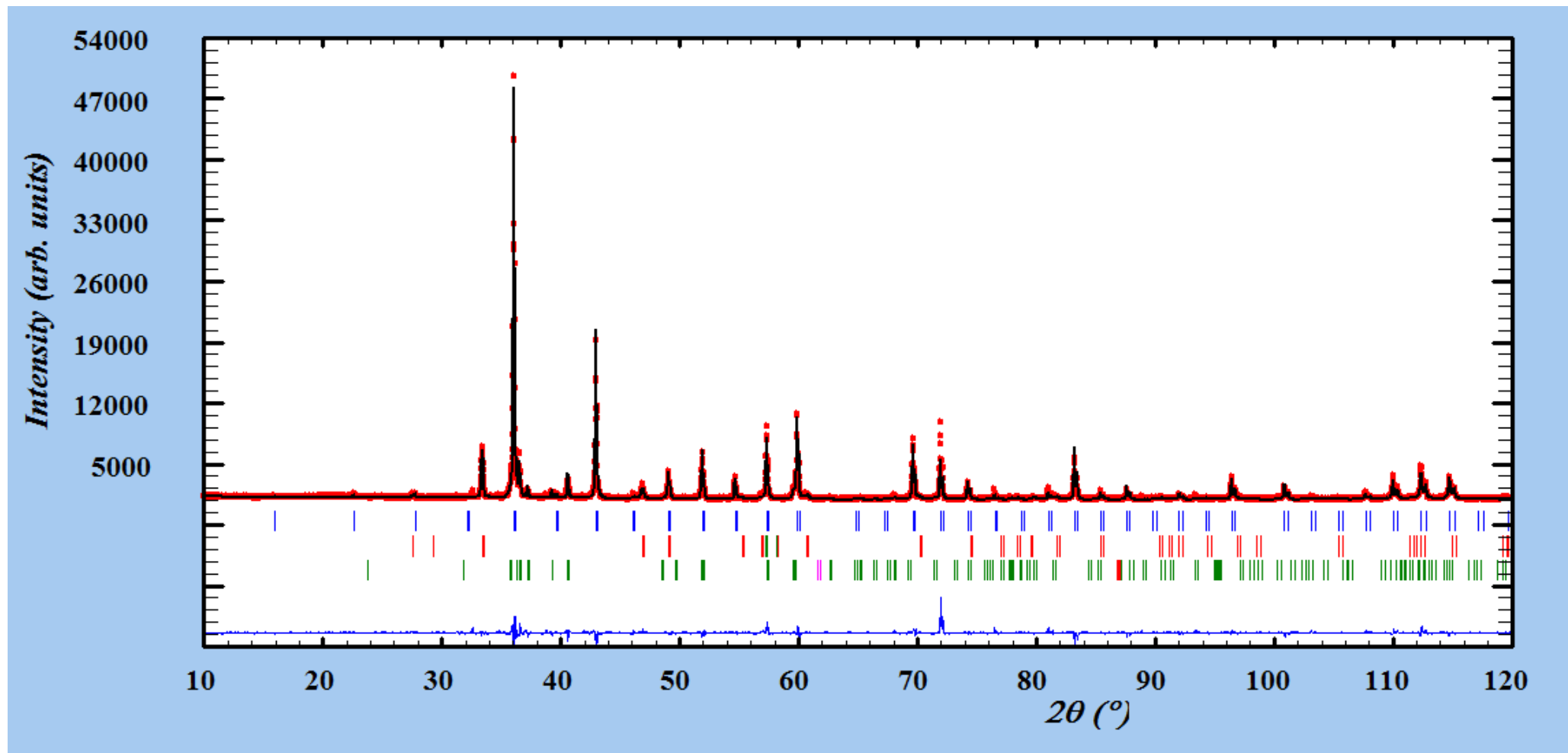

Figure S1 - Rietveld refinement plot for sample Fe100. Blue, red and green vertical lines indicate the expected peak position of skutterudite, $\mathrm{Sb}$ and $\mathrm{FeSb}_{2}$, respectively. 


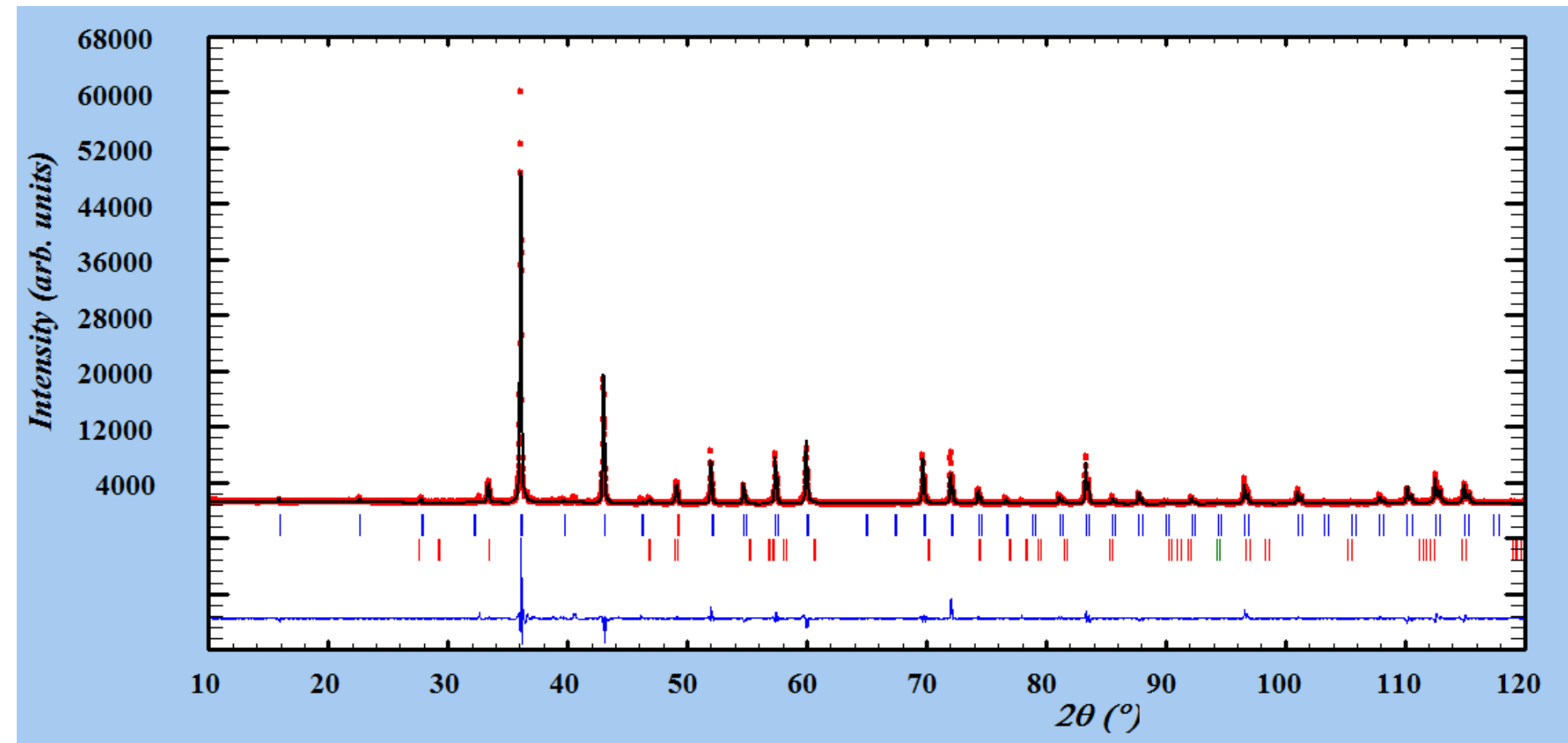

Figure S2 - Rietveld refinement plot for sample Fe90. Blue and red vertical lines indicate the expected peak position of skutterudite and $\mathrm{Sb}$, respectively. 


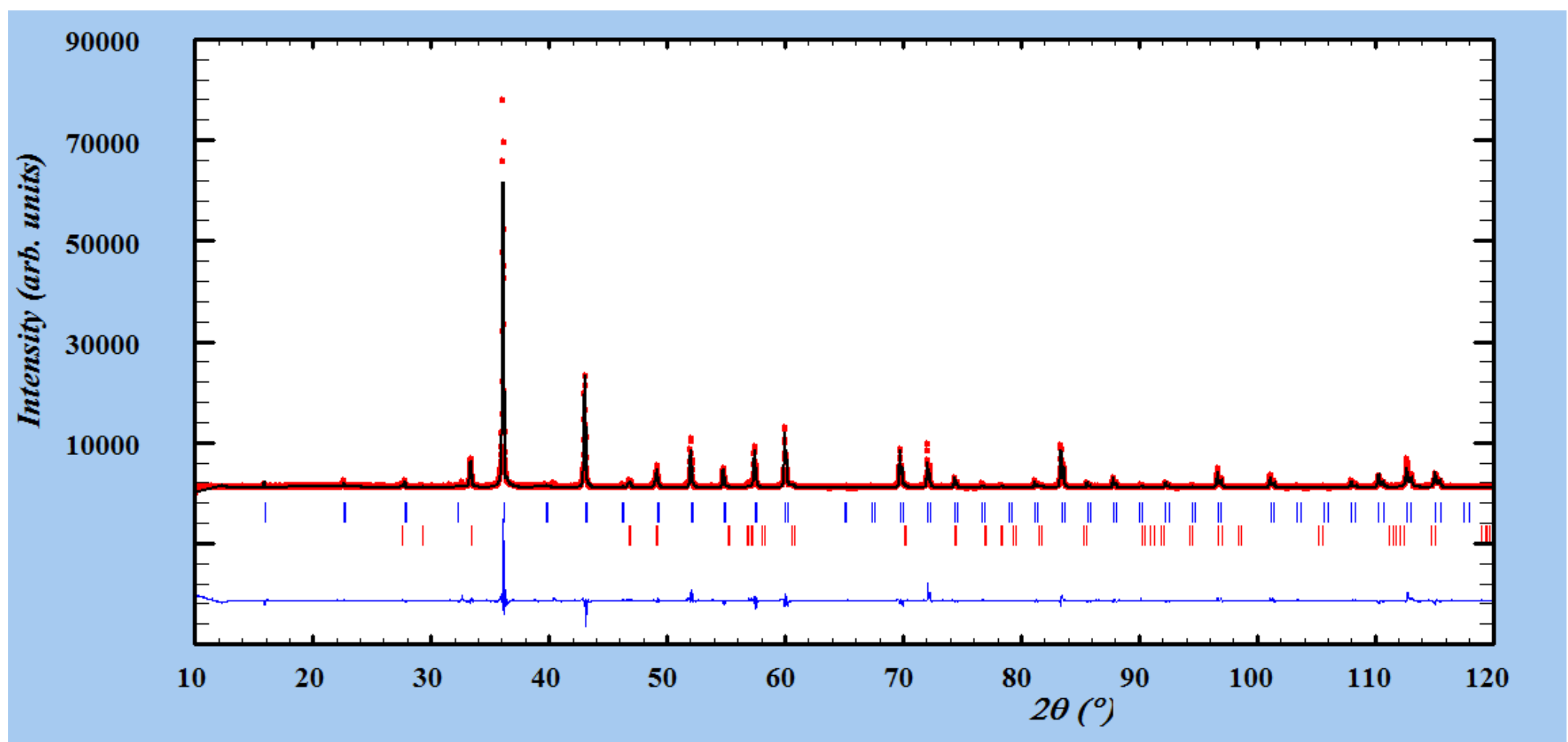

Figure S3 - Rietveld refinement plot for sample Fe80. Blue and red vertical lines indicate the expected peak position of skutterudite and $\mathrm{Sb}$, respectively. 


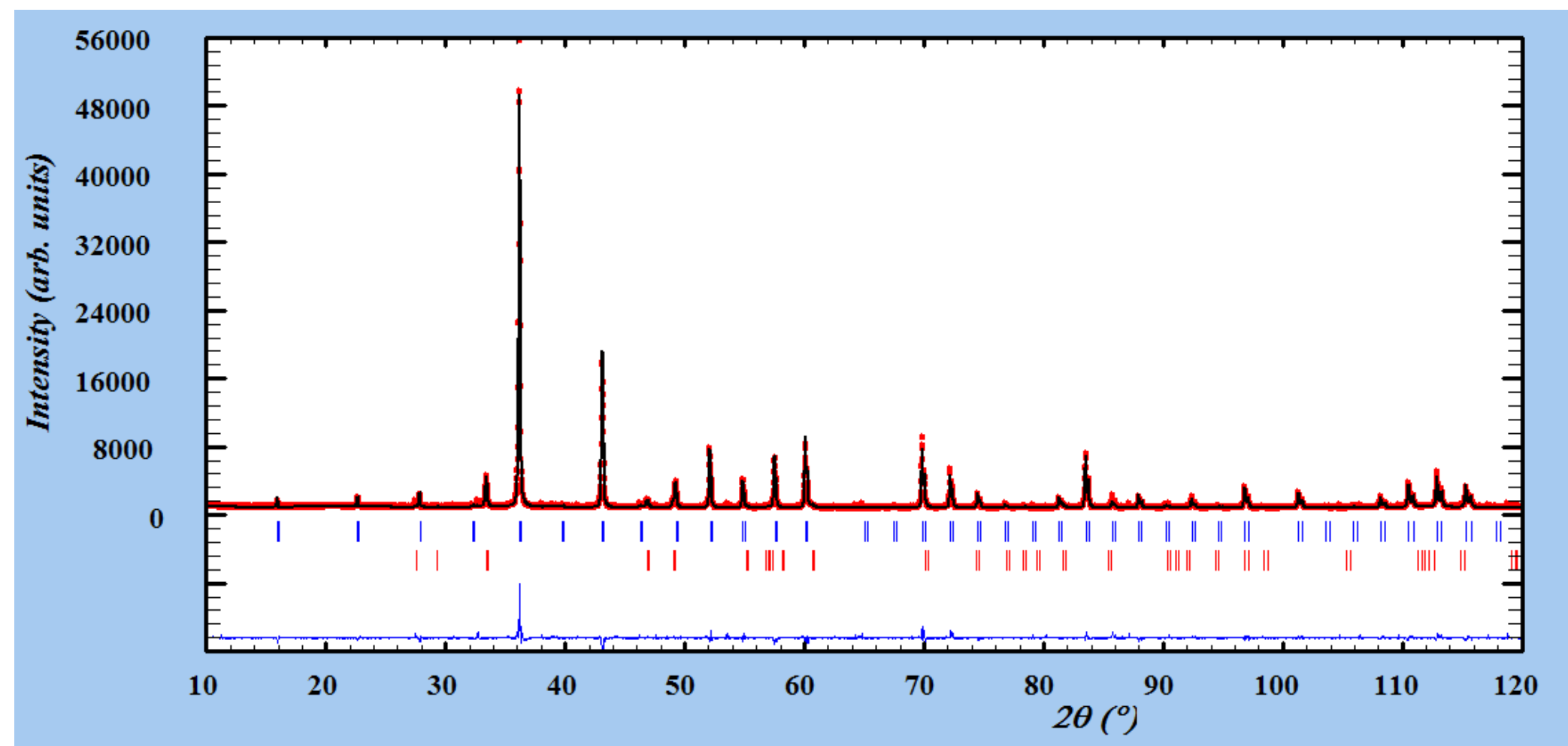

Figure S4 - Rietveld refinement plot for sample Fe70. Blue and red vertical lines indicate the expected peak position of skutterudite and $\mathrm{Sb}$, respectively. 


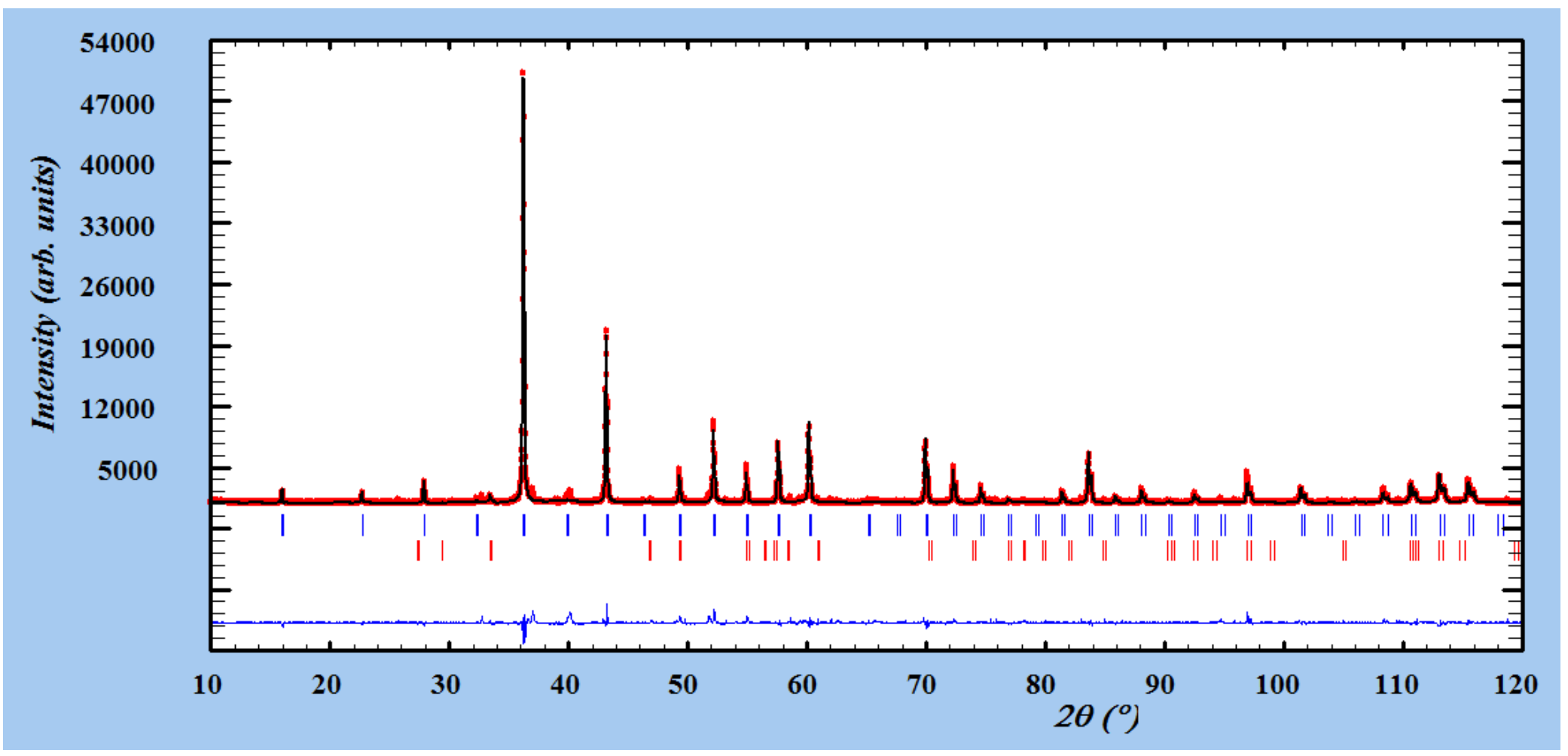

Figure S5 - Rietveld refinement plot for sample Fe63. Blue and red vertical lines indicate the expected peak position of skutterudite and $\mathrm{Sb}$, respectively. 


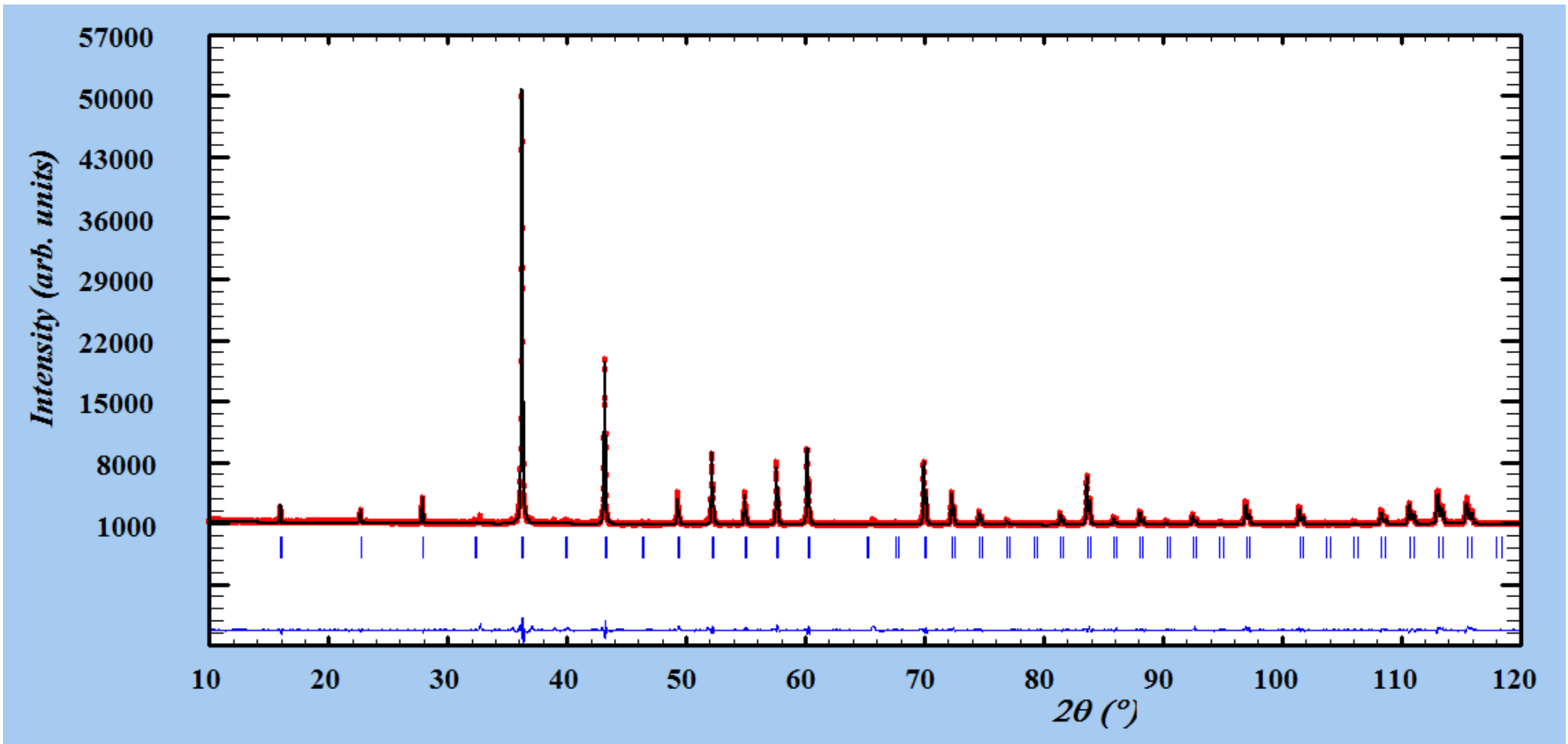

Figure S6 - Rietveld refinement plot for sample Fe60. 


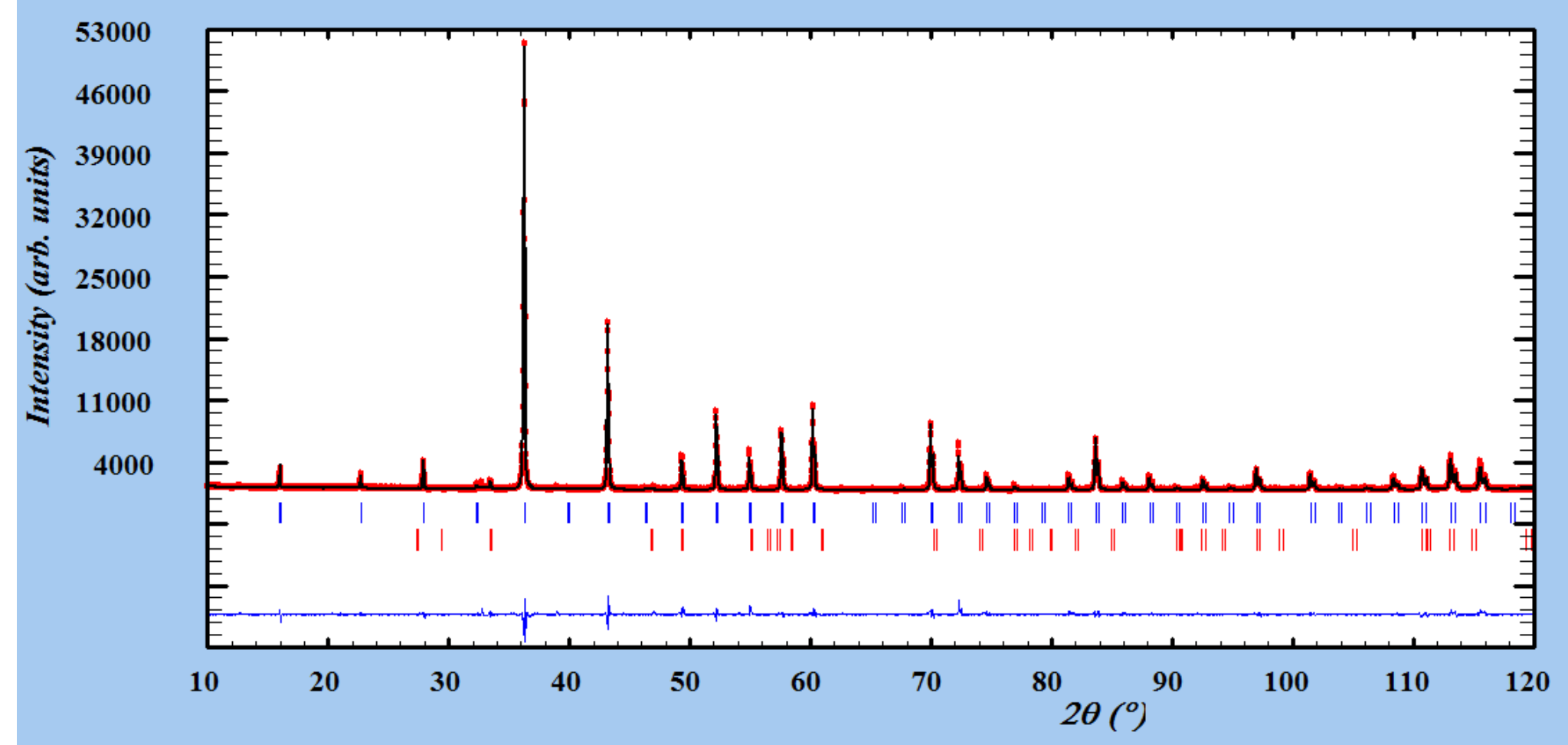

Figure S7 - Rietveld refinement plot for sample Fe58. Blue and red vertical lines indicate the expected peak position of skutterudite and $\mathrm{Sb}$, respectively. 


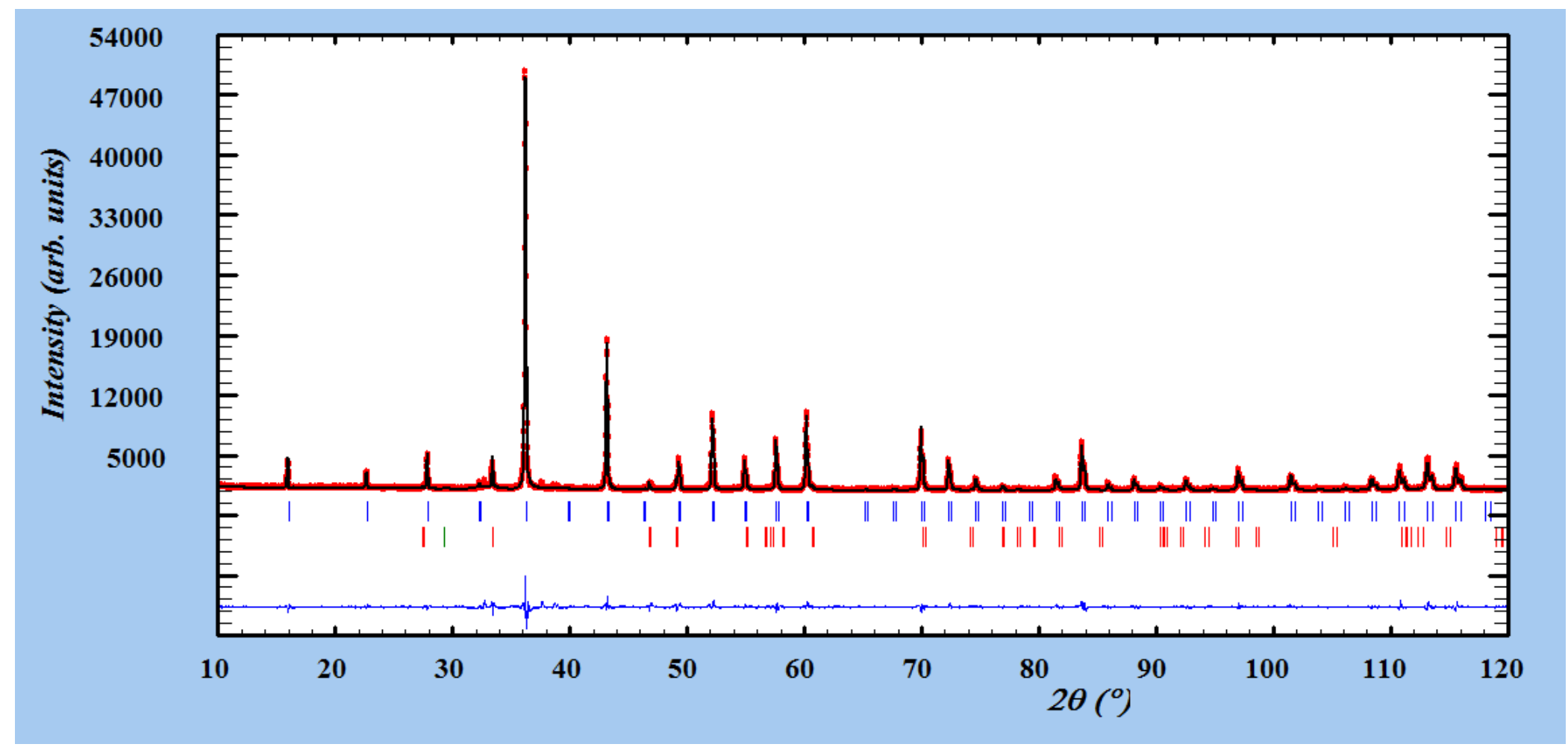

Figure S8 - Rietveld refinement plot for sample Fe55. Blue and red vertical lines indicate the expected peak position of skutterudite and $\mathrm{Sb}$, respectively. 


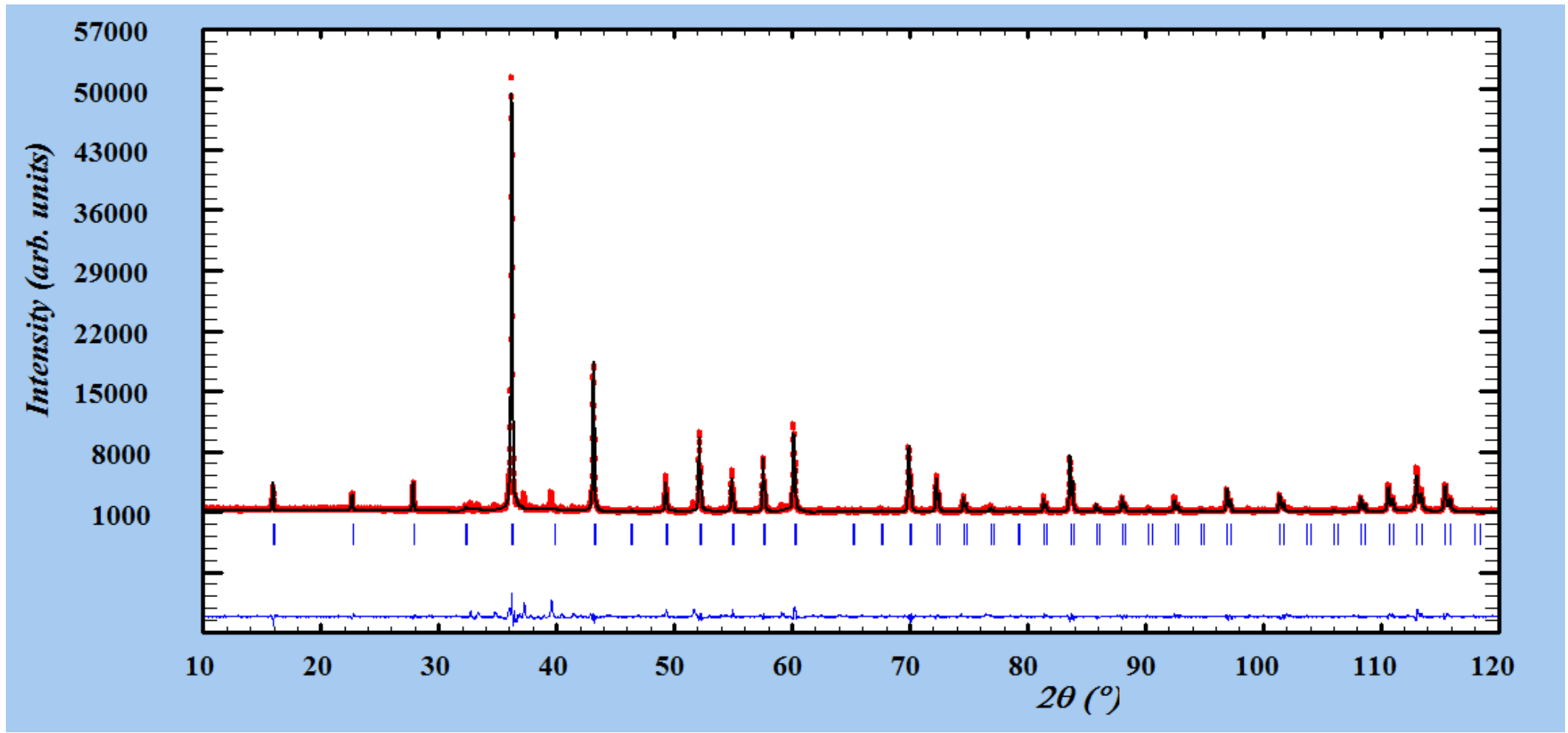

Figure S9 - Rietveld refinement plot for sample Fe50. 


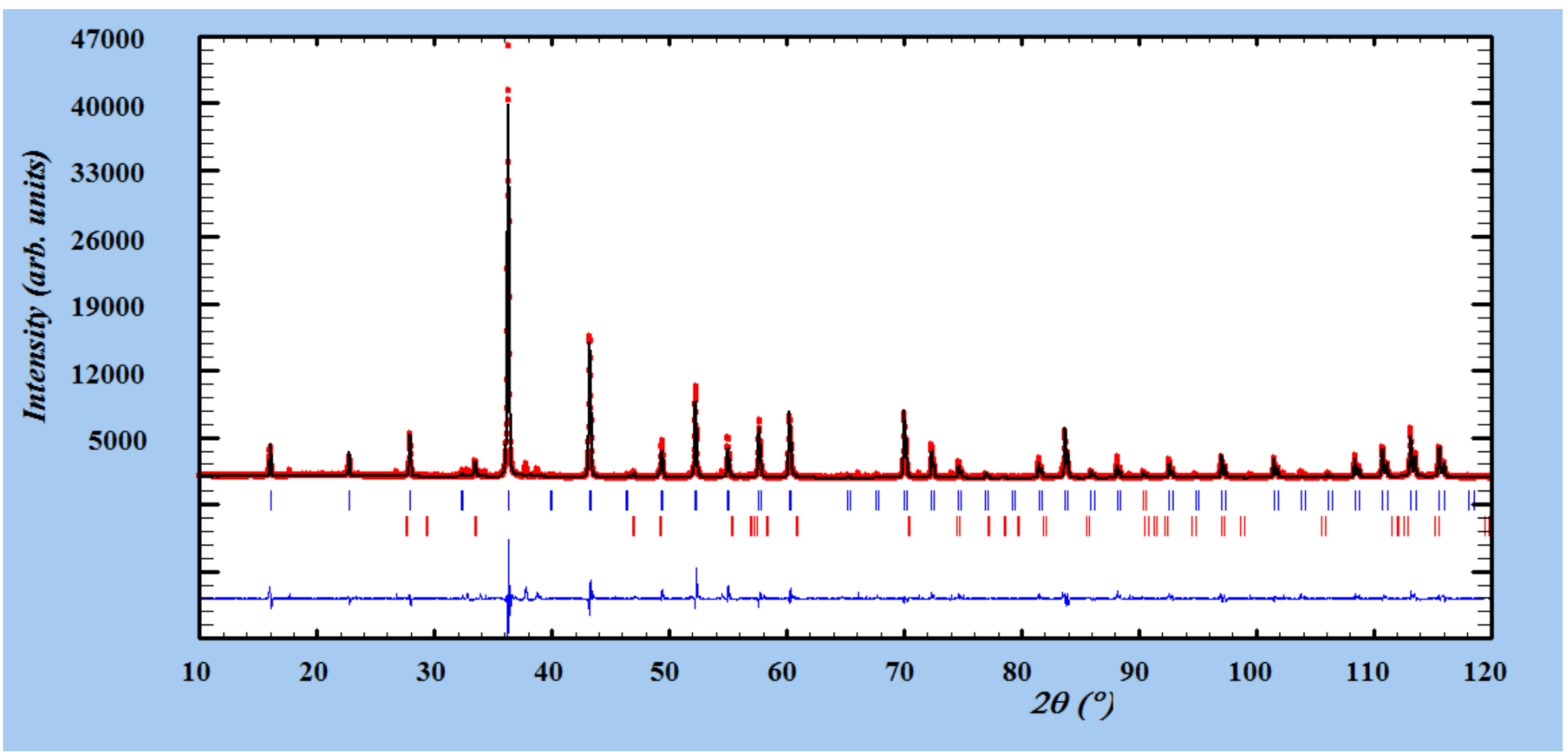

Figure S10 - Rietveld refinement plot for sample Fe40. Blue and red vertical lines indicate the expected peak position of skutterudite and $\mathrm{Sb}$, respectively. 